\title{
The SULPYCO Method Using Sulpiride Integrated with an Atypical Adjuvant Therapy for Treating Depressive Syndrome: An Observational Study
}

\section{Amgad M. Rabie*}

Pharmaceutical Organic Chemistry Department, Faculty of Pharmacy, Mansoura University, Mansoura, Dakahlia Governorate, Egypt

\begin{abstract}
In this observational study, we studied the effects of a new drug combination on depression. Patients were analyzed before and after antidepressant treatment using the Hamilton rating scale for depression (HAMD). One group of patients was treated with the new integrated medicine consisting of two separate subcutaneous injections of a low dose $(20 \mathrm{mg})$ of sulpiride and a $2.2 \mathrm{ml}$ complex homeopathic solution based on the Krebs cycle elements; each injection was administered once daily. Another group of patients was treated with conventional therapy of 20 mg sulpiride only. The third group was treated with only the homeopathic solution. The differences in the HAMD scores were evaluated before and after 3 months of treatment in these three groups of patients. The HAMD score showed a statistically significant decrease in the group treated with combined sulpiride and homeopathy. This observation suggests that a low parenteral dose $(20 \mathrm{mg})$ of sulpiride, when administered subcutaneously with a complex homeopathic remedy, may give better therapeutic results for mild and moderate depression than either sulpiride or complex homeopathy alone.
\end{abstract}

Keywords: Allohomeo; Depression; Homeopathy; Sulpiride

\section{Introduction}

Sulpiride is an atypical antipsychotic drug used mainly for the treatment of psychosis and depression [1]. At doses of over $600 \mathrm{mg}$ daily, it predominantly acts as a selective antagonist of the dopamine D2 and D3 receptors. At doses of 600-1600 mg, sulpiride is mildly sedating and antipsychotic. At low doses (100-200 mg daily), its prominent feature is antagonistic action against presynaptic inhibitory dopamine receptors, which accounts for some antidepressant activity and a stimulating effect. It also alleviates vertigo. The oral bioavailability of sulpiride is only $25-35 \%$ [2].

In Croatia, parenteral sulpiride is available at a dose of $100 \mathrm{mg}$ per vial. Oral sulpiride is available in a $50-\mathrm{mg}$ tablet dose. When used for depression, this drug is usually administered orally at a $3 \times 50 \mathrm{mg}$ daily dose [3].

This observational study analyzed patient records after treatment in order to determine whether the therapeutic action of sulpiride given parenterally by subcutaneous injection would improve if it were combined with a liquid homeopathic complex remedy based on Krebs cycle elements suitable for parenteral use. The remedy was a commercial preparation produced and sold by the German company Heel, called Coenzyme Compositum, which is available in $2.2-\mathrm{ml}$ vials and is sold as an over-the-counter drug.

\section{Material and Methods}

\section{Preparation of the combined drug}

A dose of $0.4 \mathrm{ml}(20 \mathrm{mg})$ of an isotonic solution of sulpiride was combined in two separate syringes with $2.2 \mathrm{ml}$ of an isotonic solution of mixed homeopathic substances. The dose of sulpiride was measured using a micropipette. These two injections (one with sulpiride and the other with the homeopathic remedy) were given simultaneously in the waist region using a $23 \mathrm{G}(0.6 \times 25)$ needle, once daily at $10 \mathrm{AM}$.

Homeopathic substances contained in this complex parenteral isotonic preparation were mainly compounds involved in the Krebs cycle as well as some herbal homeopathic remedies, and they were all present in equal volume:
1. Intermediates: Citric acid (D8), cis-aconitic acid (D8), alpha ketoglutaric acid (D8), succinic acid (D10), fumaric acid (D8), DL malic acid (D8), sodium diethyloxalateoacetate (D6), sodium pyruvate (D8), and barium oxalosuccinate (D10)

2. Vitamins, Nucleosides, and their Biosynthetic Intermediates: Coenzyme A (D8), nicotinamide adenine dinucleotide (NAD) (D8), adenosine triphosphate (ATP) (D10), ascorbic acid (D6), thiamine hydrochloride (D6), sodium riboflavin phosphate (D6), pyridoxine hydrochloride (D6), nicotinamide (D6), cysteine (D6), and DL-alphalipoic acid (D6)

3. Minerals: Magnesium orotate (D6), cerium oxalate (D8), and manganese phosphate (D6)

4. Herbal Extracts: Pulsatilla (D6) and Beta vulgaris (D4)

5. Miscellaneous Ingredients: Sulfur (D10) and Hepar sulfuris (D10).

The letter " $D$ " in parentheses stands for "decimal dilution", whereas the numbers that follow the " $\mathrm{D}$ " represent the number of (decimal) dilution procedures repeated according to basic homeopathy principles [4]. In this manner, "D" means that the corresponding solution of a given active homeopathic ingredient (drug) is obtained by decimal dilution of the starting mother solution. According to the German Homeopathic Pharmacopoeia published by Driehsen [5], a solution of six (6) repeated decimal dilutions in a predefined diluent-water or

*Corresponding author: Amgad M. Rabie, M.Sc., Pharm. Sci. Pharmaceutical Organic Chemistry Department, Faculty of Pharmacy, Mansoura University, Mansoura, Dakahlia Governorate, Egypt, Tel: 002-01112900494; E-mail: amgadpharmacist1@yahoo.com

Received January 02, 2013; Accepted January 29, 2013; Published January 31 2013

Citation: Rabie MA (2013) The SULPYCO Method Using Sulpiride Integrated with an Atypical Adjuvant Therapy for Treating Depressive Syndrome: An Observational Study. Adv Pharmacoepidem Drug Safety 2: 126. doi:10.4172/2167-1052.1000126

Copyright: (c) 2013 Rabie MA. This is an open-access article distributed under the terms of the Creative Commons Attribution License, which permits unrestricted use, distribution, and reproduction in any medium, provided the original author and source are credited. 
aqueous ethanol, e.g., $1 \mathrm{ml}$ of mother solution, is diluted with $9 \mathrm{ml}$ of diluent to give D1; this D1 solution $(1 \mathrm{ml})$ is subsequently diluted with $9 \mathrm{ml}$ of diluent, giving a D2 solution, etc.

After the present study, sulpiride and the complex homeopathic remedy were integrated to create a new drug, which was patented and was approved as new and inventive according to an international evaluation. Its commercial name is SULPYCO.

\section{Treatment with sulpiride alone}

In a 5-ml syringe, $0.4 \mathrm{ml}(20 \mathrm{mg})$ of isotonic sulpiride solution was combined with $2.2 \mathrm{ml}$ of isotonic $\mathrm{NaCl}$ solution. The quantity of sulpiride was measured using a micropipette.

This injection was given simultaneously in the waist region using a $23 \mathrm{G}(0.6 \times 25)$ needle, once daily at $10 \mathrm{AM}$.

\section{Treatment with only the complex homeopathic solution}

One syringe with $2.2 \mathrm{ml}$ of an isotonic solution of mixed homeopathic substances in relatively equal amounts was used.

Homeopathic substances present in this complex parenteral isotonic preparation were mainly Krebs cycle compounds as well as some herbal homeopathic remedies, all in equal volume:

1. Intermediates: Citric acid (D8), cis-aconitic acid (D8), alpha ketoglutaric acid (D8), succinic acid (D10), fumaric acid (D8), DL malic acid (D8), sodium diethyloxalateoacetate (D6), sodium pyruvate (D8), barium oxalosuccinate (D10).

2. Vitamins, Nucleosides, and Their Biosynthesis Intermediates: Coenzyme A (D8), nicotinamide adenine dinucleotide (NAD) (D8), adenosine triphosphate (ATP) (D10), ascorbic acid (D6), thiamine hydrochloride (D6), sodium riboflavin phosphate (D6), pyridoxine hydrochloride (D6), nicotinamide (D6), cysteine (D6), DL-alpha-lipoic acid (D6).

3. Minerals: Magnesium orotate (D6), cerium oxalate (D8), manganese phosphate (D6).

4. Herbal Extracts: Pulsatilla (D6) and Beta vulgaris (D4). (D10).

5. Miscellaneous Ingredients: Sulfur (D10) and Hepar sulfuris

This injection was given in the waist region using a $23 \mathrm{G}(0.6 \times 25)$ needle, once daily at $10 \mathrm{AM}$. This group was treated in the period before the first and second groups were treated.

\section{Patient groups}

The subjects of this study were 67 women, 44-80 years of age, who were suffering from depressive syndrome. One day prior to the experiment (day 0), all patients were tested using a 17 -item version of the Hamilton rating scale for depression (HAMD test). These patients came to my clinic for antidepressant treatment. Because I run a private integrated medicine clinic, they had the choice of combined treatment or only conventional or homeopathic treatment. After the treatment was completed, we analyzed the patients' HAMD scores and compared them to scores taken before the treatment, and we noticed some significant differences. These are the three patients groups that were studied:

1. The first group $(\mathrm{N}=35)$ received one dose of the combined drug (sulpiride and complex homeopathy) as two separate injections daily in the morning for 3 months.
2. The second group $(\mathrm{N}=32)$ received one dose of the single drug (sulpiride only) daily in the morning for 3 months.

3. The third group $(\mathrm{N}=15)$ received one dose of the complex homeopathy remedy as one injection daily in the morning for 3 months, but during an earlier period. At the beginning of this study, the HAMD scores in the third group were determined before and after treatment, so the data were used for statistical analysis.

The HAMD test is generally accepted as the gold standard for quantifying severity of depression symptoms such as low mood, anxiety, agitation, insomnia, and weight loss. It was performed on day 90 of taking the injections. For the 17-item version of the HAMD test, scores can range from 0 to 54 . In relation to depression, scores between 0 and 6 indicate normal personality; 7-17, mild depression; 18-24, moderate depression; $>24$, severe depression.

\section{Results}

- The average HMD score in the three groups prior to the study was $20.2 \pm 7.1$

- In the second group, the HAMD score was $18.8 \pm 9.2$ before the study and $17.3 \pm 8.8$ after the study (Table 1 ).

- In the first group, the HAMD score was $21.3 \pm 5.0$ before the study and $8.8 \pm 4.1$ after the study (Table 1 ).

- In the third group, the HAMD score was $20.7 \pm 4$ before the study and $19.4 \pm 4.5$ after the study.

The results in table 1 were subjected to a paired $t$-test. The results of the paired $t$-test, performed with the Analyse-it version 2.21 software, are shown in table 2 .

The results from the HAMD test strongly suggest that the combined therapy has a strong antidepressant activity (Table 2). The HAMD mean score decreased by 12.5 points in the first group and were far better than the results of treatment with sulpiride only and complex homeopathy.

No side effects, such as sedation, constipation, dryness of the mouth, or prolactinogenic activity, were observed.

\section{Discussion}

Sulpiride is an atypical antipsychotic drug used mainly to treat psychosis and depression. For productive psychosis, high doses of more than $600 \mathrm{mg}$ daily are used. It can be administered orally or parenterally.

\begin{tabular}{|l|l|l|}
\hline \multicolumn{2}{|c|}{ Group } & HAMD \\
\hline \multicolumn{3}{|c|}{ Before the study } \\
\hline 1 & Second group/Third group & $18.9 \pm 9.2 / 20.7 \pm 4.6$ \\
\hline 2 & First group After & $21.3 \pm 5.0$ \\
\hline \multicolumn{3}{|c|}{} \\
\hline 3 & Second group/Third group & $17.3 \pm 8.8 / 19.4 \pm 4.5$ \\
\hline 4 & First group & $8.8 \pm 4.1$ \\
\hline
\end{tabular}

Table 1: Mean HAMD score before and after therapy.

\begin{tabular}{|l|l|l|l|}
\hline Paired t-test parameters & Second group & First group & Third group \\
\hline Mean difference & 1.6 & 12.5 & 1.7 \\
\hline 95\% confidence interval (Cl) & $0.9-2.2$ & $10.9-14.2$ & $0.9-2.1$ \\
\hline Standard error (SE) & 0.31 & 0.81 & 0.32 \\
\hline$t$-statistic & 4.97 & 15.47 & 5.0 \\
\hline Degrees of freedom (DF) & 31 & 34 & 31 \\
\hline 2-tailed p value & $<0.0001$ & $<0.0001$ & $<0.0001$ \\
\hline
\end{tabular}

Table 2: Paired $t$-test results for the first and second groups. 
For psychosis with negative symptoms, long-term treatment uses moderate doses (approximately $600 \mathrm{mg}$ daily). Depression and vertigo are treated with low to moderate doses (100-200 mg daily) [1-3].

Sulpiride is absorbed slowly from the gastrointestinal tract. Its oral bioavailability is only $25-35 \%$, with marked individual differences. The peak plasma concentration is reached $4.5 \mathrm{~h}$ after oral dosing. The usual half-life is $6-8 \mathrm{~h}$. Ninety-two percent $(92 \%)$ is excreted unchanged in the urine. Sulpiride is usually given in 2 or 3 divided doses [3].

Sulpiride is a selective antagonist of the dopamine $\mathrm{D}_{2}$ and $\mathrm{D}_{3}$ receptors. This action predominates at doses exceeding $600 \mathrm{mg}$ daily. At low doses (in particular, 50-200 mg daily), its prominent feature is antagonistic action against presynaptic inhibitory dopamine receptors, which accounts for some antidepressant activity and a stimulating effect. Therefore, at these doses, it is used as a second-line antidepressant. Additionally, it alleviates vertigo [1-3]. For depression, sulpiride is given orally, at 100-200 mg daily, divided into 3 doses.

If $20 \mathrm{mg}$ of sulpiride is given subcutaneously, one part of the drug is lost in the process of injection: according to my rough estimation, only about $17.5 \mathrm{mg}$ reaches subcutaneous tissue because the process of injection results in some loss. Therefore, the overall amount of sulpiride in blood after subcutaneous injection with $20 \mathrm{mg}$ is less than $17.5 \mathrm{mg}$. In the case of a 150-mg daily dose (which is the average dose prescribed for sulpiride to treat depression), 37.5 to $52.5 \mathrm{mg}$ of the drug would be present in the blood at approximately $4.5 \mathrm{~h}$ after oral dosing [2]. In this study, I explored whether sulpiride given subcutaneously at a low dose (20 mg) would act better if combined with the complex homeopathic remedy than with isotonic $\mathrm{NaCl}$ solution.

Sulpiride was administered at a $20 \mathrm{mg}$ dose, combined either with a complex homeopathic/isopathic remedy mainly based on diluted and potentized Krebs cycle elements or with isotonic $\mathrm{NaCl}$ solution. Homeopathized (potentized) Krebs cycle components act as nonspecific metabolism activators (Witt et al. [6], according to the Reckeweg theory of isopathy and homotoxicology).

According to integrative medicine principles, we combine paradigmatically different therapeutic actions in time and space in order to possibly magnify therapeutic potential in a given patient. This is achieved by means of "synergy", which is defined as "a cooperative action of discrete agencies such that the total effect is greater than the sum of the two effects that act independently" [7].

We therefore followed the principle of integrative medicine to combine sulpiride and a complex homeopathic remedy. Two paradigmatically different substances were used together in order to multiply the therapeutic potential. We can define a "paradigm" as "a mental model, a way of seeing, a filter for one's perceptions, a frame of reference, a framework of thought or belief through which one's world or reality is interpreted, an example used to define a phenomenon, and a commonly held belief among a group of people such as scientists of a given discipline" [8]. Thomas Kuhn, a philosopher of science, says that a paradigm is a constellation of concepts, values, perceptions and practices shared by a (scientific) community that forms a particular vision of reality that is the basis of the way a (scientific) community organizes itself [8].

Conventional medicine is mainly based on a biochemical paradigm, so drugs are perceived as acting by interacting with receptors on cells. Health and disease are perceived as purely biochemical processes [9]. Although medicine strongly holds for a biochemical paradigm of biological processes, we are now in a process of revising the past century's biochemical concept. Therefore, major biological processes can also be electromagnetic in nature. Thus, we come to the concept of energy medicine, where illness is regarded also and at the same time as a disturbance in energy fields and can be addressed via interventions into those energies and energy fields [9]. The paradigm shift, as a change from one way to another, is not a transformative revolution but a sort of gradual metamorphosis driven by agents of small bits of slow change [8], and integrative medicine is surely one of those small bits.

Consequently, sulpiride acts at a biochemical level or paradigm, while homeopathic medicines surely do not, since the quantity of diluted matter is so small that it cannot satisfy the receptor theory in a biochemical paradigm $[10,11]$. There are disputes about the efficiency of homeopathic medicine. Although many suppose that homeopathy works on a placebo principle, others hold a different opinion [12-14] Nevertheless, even though we do not fully understand how homeopathy works, it is usually perceived as being energetically programmed water interacting with water in the body, which affects cells on an energetic (quantum field) level [13]. This is the proposed mechanism of action for high dilutions/potencies, which integrate global dynamics also by electromagnetic regulation.

Another model emerging from the nonlinear complex systems theory has been proposed for low potencies [7]. A quantum and nonlinear physical model for homeopathy may work in concordance as well, so at the same time both mechanisms of action may be in play.

The findings of the present study indicate that sulpiride at a low dose, given subcutaneously in combination with a complex homeopathic remedy, acts better than sulpiride with isotonic $\mathrm{NaCl}$ solution does.

My opinion is that the body exists on different levels or in different paradigms that are mutually related concurrently. Therefore, we cannot say definitively that the body is just a machine or just a computer or just a quantum operator. It is all of these at the same time. For example, if a bone is fractured, it should first be treated mechanically, i.e., operated on with osteosynthesis (mechanical paradigm). In order for it to be healed, growing processes (biochemical paradigm) should be applied. If we apply magnetic therapy to a fracture site, it can grow even faster (energetic paradigm), so by combining mechanical, biochemical and energetic processes, we may achieve positive synergy and multiply the healing potential. Thus, we see that the body is a complex system, which means at least two things:

1. It is a system composed of interconnected parts that, as a whole, exhibits one or more properties that are not obvious from the properties of the individual parts; the whole is more than merely the sum of its parts.

2. It is also a network of heterogeneous components that interact nonlinearly. In a linear system, an effect is always directly proportional to cause, whereas in a complex system, a small perturbation may cause a large effect (the butterfly effect), a proportional effect, or even no effect at all. Here, we come to the principle of the chaos theory [7].

In the context of this study, I speculate that a homeopathic remedy can make a small change in cellular energy production so that sulpiride can perform better and at a smaller dose. The foremost problem with sulpiride in low doses is a strong stimulation of prolactin secretion; whether this may contribute to the development of breast cancer in women is currently not known, but in this study, no milk production or breast stimulation was observed.

Whether such combined allohomeo (R) therapy is really a therapeutic possibility needs to be investigated in further studies, and it 
Citation: Rabie MA (2013) The SULPYCO Method Using Sulpiride Integrated with an Atypical Adjuvant Therapy for Treating Depressive Syndrome: An Observational Study. Adv Pharmacoepidem Drug Safety 2: 126. doi:10.4172/2167-1052.1000126

Page 4 of 4

will have paramount significance. It would enable us to reduce the dose of chemical drugs, thereby helping to avoid drug side effects while still achieving the desired therapeutic effect.

\section{Conclusion}

This study confirms that sulpiride at a low dose $(20 \mathrm{mg})$ taken parenterally has a significantly better effect on depression if combined with a complex homeopathic/isopathic remedy based on Krebs cycle components than if combined with an isotonic solution of $\mathrm{NaCl}$. It also presupposes that homeopathy does not work on the placebo system. Further experiments are necessary to determine whether this speculation is valid.

\section{References}

1. Komossa K, Depping AM, Gaudchau A, Kissling W, Leucht S (2010) Secondgeneration antipsychotics for major depressive disorder and dysthymia. Cochrane Database Syst Rev 12: CD008121.

2. Martí Massó JF, Ruiz-Martínez J, Bergareche A, López de Munain A (2011) Parkinsonism induced by sulpiride and veralipride: two different stories. Med Clin (Barc) 137: 473-474.

3. Jo SH, Lee SY (2010) Response of $\mathrm{i}(\mathrm{kr})$ and HERG currents to the antipsychotics tiapride and sulpiride. Korean J Physiol Pharmacol 14: 305-310.

4. Weingarther $O(2007)$ The nature of the active ingredient in ultramolecular dilutions. Homeopathy 96: 220-226.

5. Driehsen W (2003) German Homeopathic Pharmacopoeia, (5thedn), Gauteng, South Africa, Medpharm, November 3, 2003.
6. Witt CM, Bluth M, Albrecht H, Weisshuhn TE, Baumgartner S, et al. (2007) The in vitro evidence for an effect of high homeopathic potencies-a systematic review of the literature. Complement Ther Med 15: 128-138.

7. Rocha LM (1999) Complex Systems Modeling: Using Metaphors From Nature in Simulation and Scientific Models. BITS: Computer and Communications News. Computing, Information, and Communications Division. Los Alamos National Laboratory. November 1999.

8. Kuhn TS (1965) The structure of Scientific Revolutions, (2ndedn) University of Chicago Press, Chicago, IL,U.S.A.

9. Becker RO (2004) Exploring new horizons in electromedicine. J Altern Complement Med 10: 17-18.

10. Taylor MA, Reilly D, Llewellyn-Jones RH, McSharry C, Aitchison TC (2000) Randomised controlled trial of homoeopathy versus placebo in perennial allergic rhinitis with overview of four trial series. BMJ 321: 471-476.

11. Bell IR, Lewis DA 2nd, Brooks AJ, Schwartz GE, Lewis SE, et al. (2004) Improved clinical status in fibromyalgia patients treated with individualized homeopathic remedies versus placebo. Rheumatology (Oxford) 43: 577-582.

12. Linde K, Clausius N, Ramirez G, Melchart D, Eitel F, et al. (1997) Are the clinical effects of homeopathy placebo effects? A meta-analysis of placebo-controlled trials. Lancet 350: 834-843.

13. Vickers AJ, Smith C (2009) Homeopathic Oscillococcinum for preventing and treating influenza and influenza-like syndromes. Cochrane Database Syst Rev CD001957.

14. Lüdtke R, Rutten AL (2008) The conclusions on the effectiveness of homeopathy highly depend on the set of analyzed trials. J Clin Epidemiol 61: 1197-1204. 